\title{
PREFERRED OPTIMAL STRATEGIES
}

\author{
R. C. $\mathrm{BUCK}^{1}$
}

Let $\Gamma$ be the normalized two person zero sum game defined by a pay-off function $M(x, y)$, for $x \in A, y \in B$. If $A$ and $B$ are compact convex sets in a finite dimensional space, and $M$ is bilinear, then $\Gamma$ is strictly determined. Then $\Gamma$ has a value $v(\Gamma)$, and the players have optimal strategy sets $A_{1} \subset A, B_{1} \subset B$, such that $M\left(x, y_{1}\right) \leqq v(\Gamma)$ $\leqq M\left(x_{1}, y\right)$ for any choices of $x \in A, x_{1} \in A_{1}, y \in B, y_{1} \in B_{1}$. We may denote the game $\Gamma$ by $\langle M, A, B\rangle$. (See $[1 ; 2]$.)

This note is concerned with games in which the first player $P_{1}$ has more than one optimal strategy. Since $A_{1}$ is convex, there are then an infinite number. Against an optimal strategy of $P_{2}$, none of these will achieve more than $v(\Gamma)$. However, if $P_{2}$ should play nonoptimally, $P_{1}$ might obtain more than $v(\Gamma)$, and the outcome might depend upon which optimal strategy from the set $A_{1}$ he chooses. In many applications of game theory, it is desirable to have a systematic procedure for choosing a preferred strategy $\bar{x}$ in $A_{1}$ which will take advantage of the possibility of error (nonintelligent action) on the part of the second player. Such a procedure will be given in this note; the resulting preferred optimal strategy is unique, up to equivalence, when the set $B$ is a polyhedron.

Two first player strategies, $x^{\prime}$ and $x^{\prime \prime}$, are said to be equivalent for the same $\Gamma$ if $M\left(x^{\prime}, y\right)=M\left(x^{\prime \prime}, y\right)$ for all $y \in B$. When $B$ is polyhedral, it has only a finite number of extreme points $\pi$. These we call "pure" strategies for $P_{2}$. Any $y \in B$ is then a finite convex combination of pure strategies. We divide the pure strategies of the second player into two classes. A pure strategy $\pi$ is good if it is present in at least one optimal $P_{2}$ strategy (i.e. if it occurs with nonzero coefficient in an optimal strategy). All other pure strategies are called poor. The dichotomy can also be made analytically. If $\pi$ is a good pure strategy, then $M\left(x_{1}, \pi\right)=v(\Gamma)$ for every optimal $x_{1} \in A_{1}$; if $\pi$ is a poor pure strategy, then there is at least one $x_{1} \in A_{1}$ with $M\left(x_{1}, \pi\right)>v(\Gamma)$. Let $B^{(1)}$ be the closed convex hull of the set of poor pure strategies of $P_{2}$.

THEOREM 1. The following statements are equivalent: (i) $B^{(1)}$ is void, (ii) $B_{1}$ contains a point interior to $B$, (iii) all the strategies in $A_{1}$ are equivalent.

Received by the editors August 28, 1957.

1 This work was partially supported by the Office of Ordnance Research, U. S. Army. 
Let $B_{*}$ be the set of all points $y \in B$ such that $M(x, y)=v(\Gamma)$ for every optimal $x \in A_{1}$. This set is convex and contains $B_{1}$, but is disjoint from $B^{(1)}$. Moreover, any line segment in $B$ which contains a point of $B_{*}$ in its interior, lies wholly in $B_{*}$; thus, $B_{*}$ is the convex hull of the set of good pure strategies. When (ii) holds, $B=B_{*}$. When (ii) fails, $B_{*}$ is the face of $B$ containing $B_{1}$, and $B \neq B_{*}$. Finally, it is immediate that (i) and (iii) are each equivalent to $B=B_{*}$.

Construct a new game, $\Gamma_{1}=\left\langle M, A_{1}, B^{(1)}\right\rangle$. To this, we may apply the same procedure, generating a sequence of games $\Gamma_{1}, \Gamma_{2}, \Gamma_{3} \ldots$ with $\Gamma_{n}=\left\langle M, A_{n}, B^{(n)}\right\rangle$. We have $A \supset A_{1} \supset A_{2} \supset \cdots$ and $B \supset B^{(1)}$ $\supset B^{(2)} \supset \cdots ; A_{n+1}$ is the set of optimal strategies for $P_{1}$ in the game $\Gamma_{n}$, and $B^{(n+1)}$ is the convex hull of the poor pure strategies for $P_{2}$ in $\Gamma_{n}$. Moreover, the vertices of $B^{(n+1)}$ form a proper subset of those of $B^{(n)}$. When $B$ is polyhedral, having a finite number of vertices, we must reach an integer $N$ such that $B^{(N+1)}$ is void. In the game $\Gamma_{N}$, $P_{2}$ will have no poor pure strategies. By Theorem 1 , then, all of the optimal strategies $\bar{x}$ in the set $A_{\infty}=A_{N+1}$ are then equivalent in the game $\Gamma_{N}$.

\section{Theorem 2. The strategies $\bar{x}$ in $A_{\infty}$ are all equivalent in $\Gamma$.}

Let $\bar{x} \in A_{\infty}$. Then, $\bar{x} \in A_{n}$ for any $n$. If $\pi$ is any extreme point of $B$ which is a good strategy for $P_{2}$ in $\Gamma_{n}$, then $M(\bar{x}, \pi)=v\left(\Gamma_{n}\right)$. Every extreme point $\pi$ is good in $\Gamma$, or in one of the games $\Gamma_{j}$. Thus, $M(\bar{x}, \pi)$ $=M(x, \pi)$, for every $\pi$ and any choice of $x \in A_{\infty}$. Since $B$ is the convex hull of the points $\pi, M(\bar{x}, y)=M(x, y)$ for every $y \in B$, and any choice of $x \in A_{\infty}$. Thus, all of the points of the set $A_{\infty}$ are equivalent in $\Gamma$.

By this process, then, we have arrived at a strategy $\bar{x}$ which is optimal in each of the games $\Gamma, \Gamma_{1}, \Gamma_{2}, \cdots$, and which (when $B$ is polyhedral) is unique, up to equivalence. When $B$ is not polyhedral, the sequence $B^{(n)}$ may not terminate. However, the strategies in the set $A_{\infty}$ still have the desirable properties described above, and are preferred optimal strategies.

We give a simple illustration. Consider the rectangular game whose (discrete) pay off matrix is

$$
W=\left[\begin{array}{lllll}
6 & 1 & 5 & 8 & 5 \\
2 & 7 & 3 & 4 & 4 \\
6 & 1 & 7 & 4 & 5
\end{array}\right] .
$$

If $\Gamma$ is the mixed game derived from $W$, so that $A$ is a triangle and $B$ a 4 -simplex, then $v(\Gamma)=4, P_{1}$ has two basic (extreme) optimal strategies $x^{\prime}=(1 / 2,1 / 2,0), x^{\prime \prime}=(0,1 / 2,1 / 2)$ and $P_{2}$ has a unique optimal 
strategy $y=(3 / 5,2 / 5,0,0,0)$. The poor pure strategies for $P_{2}$ are columns 3,4 and 5 .

Proceeding as above, the game $\Gamma_{1}$ is then the mixed game obtained from the rectangular matrix

$$
W=\left[\begin{array}{lll}
4 & 6 & 9 / 2 \\
5 & 4 & 9 / 2
\end{array}\right]
$$

This was obtained by computing $\left[x^{\prime}, x^{\prime \prime}\right] W$, and deleting from this matrix the first two columns. The value of $\Gamma_{1}$ is $v\left(\Gamma_{1}\right)=9 / 2$, and $P_{1}$ has the optimal strategies $(1 / 2,1 / 2)$ and $(1 / 4,3 / 4)$. The last column is optimal for $P_{2}$. Repeating the process, $\Gamma_{2}$ is the mixed game obtained from

$$
W=\left[\begin{array}{cc}
9 / 2 & 5 \\
19 / 2 & 9 / 2
\end{array}\right] .
$$

We see that $v\left(\Gamma_{2}\right)=14 / 3$, that $P_{1}$ has a unique optimal strategy $(1 / 3,2 / 3)$, and that $B^{(2)}$ is empty. Retracing our steps, we arrive at $\bar{x}=(1 / 6,1 / 2,1 / 3)$ which is the unique preferred optimal strategy. Note that its pay-off is $\bar{x} W=(4,4,14 / 3,14 / 3,9 / 2)$.

\section{REFERENCES}

1. J. McKinsey, Introduction to the theory of games, McGraw-Hill, 1952.

2. - Contributions to the theory of games I, II, Math. Study 24, 28, Princeton University Press, 1950, 1953.

UNIVERSITY OF WiSCONSIN 